\title{
Bounds on Isocurvature Perturbations from Cosmic Microwave Background and Large Scale Structure Data
}

\author{
Patrick Crotty, ${ }^{1}$ Juan García-Bellido, ${ }^{2}$ Julien Lesgourgues, ${ }^{1,3}$ and Alain Riazuelo ${ }^{4}$ \\ ${ }^{1}$ Laboratoire de Physique Théorique LAPTH, F-74941 Annecy-le-Vieux Cedex, France \\ ${ }^{2}$ Departamento de Física Teórica C-XI, Universidad Autónoma de Madrid, Cantoblanco, 28049 Madrid, Spain \\ ${ }^{3}$ TH-Division CERN, CH-1211 Gèneve 23, Switzerland \\ ${ }^{4}$ Service de Physique Théorique CNRS, CEA/Saclay F-91191, Gif-sur-Yvette Cedex, France
}

(Received 17 June 2003; published 24 October 2003)

\begin{abstract}
We obtain very stringent bounds on the possible cold dark matter, baryon, and neutrino isocurvature contributions to the primordial fluctuations in the Universe, using recent cosmic microwave background and large scale structure data. Neglecting the possible effects of spatial curvature, tensor perturbations, and reionization, we perform a Bayesian likelihood analysis with nine free parameters, and find that the amplitude of the isocurvature component cannot be larger than about $31 \%$ for the cold dark matter mode, $91 \%$ for the baryon mode, $76 \%$ for the neutrino density mode, and $60 \%$ for the neutrino velocity mode, at $2 \sigma$, for uncorrelated models. For correlated adiabatic and isocurvature components, the fraction could be slightly larger. However, the cross-correlation coefficient is strongly constrained, and maximally correlated/anticorrelated models are disfavored. This puts strong bounds on the curvaton model.
\end{abstract}

Introduction. - Thanks to the tremendous developments in observational cosmology during the last few years, it is possible to speak today of a standard model of cosmology, whose parameters are known within systematic errors of just a few percent. Moreover, the recent measurements of both temperature and polarization anisotropies in the cosmic microwave background (CMB) have opened the possibility to test not only the basic paradigm for the origin of structure, namely, inflation, but also the precise nature of the primordial fluctuations that gave rise to the CMB anisotropies and the density perturbations responsible for the large scale structure (LSS) of the Universe.

The simplest realizations of the inflationary paradigm predict an approximately scale invariant spectrum of adiabatic and Gaussian curvature fluctuations, whose amplitude remains constant outside the horizon, and therefore allows cosmologists to probe the physics of inflation through observations of the CMB anisotropies and the LSS matter distribution. However, this is by no means the only possibility. Multiple-field inflation predicts that, together with the adiabatic component, there should also be an entropy or isocurvature perturbation [1-3] associated with fluctuations in number density between different components of the plasma before decoupling, with a possible statistical correlation between the adiabatic and isocurvature modes [4]. Baryon and cold dark matter (CDM) isocurvature perturbations were proposed long ago [5] as an alternative to adiabatic perturbations. Recently, two other modes, neutrino isocurvature density and velocity perturbations, have been added to the list [6]. Moreover, it is well known that entropy perturbations seed curvature perturbations outside the horizon [2,3], so it is possible that a significant component of the observed adiabatic mode could be strongly correlated with an isocurvature mode. Such models are generically

171301-1

0031-9007/03/91(17)/171301(4)\$20.00 called curvaton models [7,8], and are now widely studied as an alternative to the standard paradigm. Furthermore, isocurvature modes typically induce non-Gaussian signatures in the spectrum of primordial perturbations.

In this Letter we present very stringent bounds on the various isocurvature components, coming from the temperature power spectrum (TT) and temperaturepolarization cross correlation (TE) recently measured by the Wilkinson Microwave Anisotropy Probe (WMAP) satellite [9], from the small-scale temperature anisotropy probed by ACBAR [10], and from the matter power spectrum measured by the 2-degree-Field Galaxy Redshift Survey (2dFGRS) [11]. We do not use the data from Lyman- $\alpha$ forests, since they are based on nonlinear simulations carried under the assumption of adiabaticity. We will not assume any specific model of inflation, or any particular mechanism to generate the perturbations (late decays, phase transitions, cosmic defects, etc.), and thus will allow all five modes - adiabatic (AD), baryon isocurvature (BI), CDM isocurvature (CDI), neutrino isocurvature density (NID), and neutrino isocurvature velocity (NIV) - to be correlated (or not) among each other and to have arbitrary tilts. However, we will consider only the mixing of the adiabatic mode and one of the isocurvature modes at a time. This choice has the advantage of restricting the number of free parameters and takes into account the fact that most of the proposed mechanisms for the generation of isocurvature perturbations lead to only one mode. The first bounds on isocurvature perturbations assumed uncorrelated modes [12], but recently also correlated ones were considered [13-16].

The present analysis neglects the possible effects of spatial curvature, tensor perturbations, and reionization. Therefore, each model is described by nine parameters: the cosmological constant $\Omega_{\Lambda}$, the baryon density $\omega_{\mathrm{B}}=$ $\Omega_{\mathrm{B}} h^{2}$, the cold dark matter density $\omega_{\text {cdm }}=\Omega_{\text {cdm }} h^{2}$, the

(C) 2003 The American Physical Society

171301-1 
overall normalization $A$, the isocurvature mode relative amplitude $\alpha$ and correlation $\beta$, the adiabatic and isocurvature tilts $\left(n_{\mathrm{ad}}, n_{\text {iso }}\right)$, and finally a free bias $b$ associated with the $2 \mathrm{dF}$ power spectrum. We generate a five-dimensional grid of models $(A, \alpha, \beta$, and $b$ are not discretized) and perform a Bayesian analysis in the full nine-dimensional parameter space. At each grid point, we store some $C_{l}$ values in the range $0<l<1800$ and some $P(k)$ values in the range probed by the $2 \mathrm{dF}$ data. The likelihood of each model is then computed using the software or the detailed information provided on the experimental websites, using 1398 points from WMAP, 11 points from ACBAR, and 32 points from the 2dFGRS. For parameter values which do not coincide with grid points, our code first performs a cubic interpolation of each power spectrum, accurate to better than $1 \%$, and then computes the likelihood of the corresponding model. From the limitation of our grid, we impose the flat prior $n_{\text {iso }}>0.6$ (as preferred by inflation). The other grid ranges are wide enough in order not to affect our results.

For the theoretical analysis, we will use the notation and some of the approximations of Ref. [14]. For instance, the power spectra of adiabatic and isocurvature perturbations, as well as their cross correlation, are parametrized with three amplitudes and two spectral indices,

$$
\begin{aligned}
\Delta_{\mathcal{R}}^{2}(k) & \equiv \frac{k^{3}}{2 \pi^{2}}\left\langle\mathcal{R}^{2}\right\rangle=\mathcal{A}^{2}\left(\frac{k}{k_{0}}\right)^{n_{\mathrm{ad}}-1}, \\
\Delta_{S}^{2}(k) & \equiv \frac{k^{3}}{2 \pi^{2}}\left\langle S^{2}\right\rangle=\mathcal{B}^{2}\left(\frac{k}{k_{0}}\right)^{n_{\mathrm{iso}}-1}, \\
\Delta_{\mathcal{R} S}^{2}(k) & \equiv \frac{k^{3}}{2 \pi^{2}}\langle\mathcal{R} S\rangle=\mathcal{A} \mathcal{B} \cos \Delta\left(\frac{k}{k_{0}}\right)^{\left(n_{\mathrm{ad}}+n_{\mathrm{iso}}\right) / 2-1},
\end{aligned}
$$

where $k_{0}$ is an arbitrary pivot scale. We also assume that the correlation coefficient $\cos \Delta$ is scale independent (note that in Ref. [16] this assumption was relaxed). In order to evaluate the temperature and polarization anisotropies, one has to calculate the radiation transfer functions for adiabatic and isocurvature perturbations and compute the total angular power spectrum as [14]

$$
C_{l}=C_{l}^{\mathrm{ad}}+B^{2} C_{l}^{\text {iso }}+2 B \cos \Delta C_{l}^{\text {corr }},
$$

where $B$ is the entropy to curvature perturbation ratio during radiation domination, $B=S / \mathcal{R}$. We will use here a slightly different notation, used before by other groups $[4,12]$, where

$$
C_{l}=(1-\alpha) C_{l}^{\mathrm{ad}}+\alpha C_{l}^{\mathrm{iso}}+2 \beta \sqrt{\alpha(1-\alpha)} C_{l}^{\mathrm{corr}} .
$$

The two notations are related by

$$
\alpha=B^{2} /\left(1+B^{2}\right), \quad \beta=\cos \Delta .
$$

This notation has the advantage that the full parameter space of $[\alpha, 2 \beta \sqrt{\alpha(1-\alpha)}]$ is contained within a circle of radius $1 / 2$. The North and South rims correspond to fully correlated $(\beta=+1)$ and fully anticorrelated $(\beta=-1)$ perturbations, with the equator corresponding to uncorrelated perturbations $(\beta=0)$. The East and West correspond to purely isocurvature and purely adiabatic perturbations, respectively. Any other point within the circle is a particular admixture of adiabatic and isocurvature modes.

In order to compute the $C_{l}$ coefficients of the temperature and polarization power spectra, as well as the matter spectra $P(k)$, we have used a CMB code developed by one of us (A. R.) which coincides, within $2 \%$ errors, with the values provided by CMBFAST for $\mathrm{AD}$ and CDI modes, and also includes the neutrino isocurvature modes as well as the cross-correlated power spectra. Note that the code defines the tilt of each power spectrum with respect to a pivot scale $k_{0}$ corresponding the present value of the Hubble radius, while CMBFAST uses $k_{0}=0.05 \mathrm{Mpc}^{-1}$ : so, the comparison of our results with those of Ref. [9] for the CDI mixed model is not straightforward.

Results. - We now describe the different bounds, summarized in Table I. For each specific mode, the probability distribution for $\alpha$ (uncorrelated case) and the likelihood contours in the $(\alpha, \beta)$ plane (correlated case) are shown in Fig. 1. The best-fitting parameter set for each model is given in Table II.

CDM isocurvature.-Assuming no correlation between the adiabatic and CDI modes, we find an upper bound $\alpha<0.31$ at $95 \%$ C.L. The deviation from a pure adiabatic model does not improve the goodness of fit, since the minimum $\chi^{2}$ value goes from 1478.8 to 1478.3 , while the number of degrees of freedom decreases from 1435 to 1433 . The situation does not change significantly when we include a possible correlation: the minimum $\chi^{2}$ is still around 1478.0, and the individual $2 \sigma$ bounds on the coefficients read $\alpha<0.47$ and $-0.31<2 \beta \sqrt{\alpha(1-\alpha)}<$ 0.31 at $95 \%$ C.L. Most of the constraints on this model come from the WMAP TT power spectrum. For comparison, we repeated our analysis replacing WMAP and ACBAR by the Wang et al. [17] CMB data compilation, which includes all the pre-WMAP temperature data. In that case, the results exhibit a wide parameter degeneracy (as indicated by previous studies $[13,14]$ ), and a large fraction of anticorrelated isocurvature modes cannot be excluded, up to $(\alpha, \beta)=(0.9,-1)$, provided one allows for a very large baryon fraction. Therefore, we conclude that the WMAP TT data are very powerful in constraining the isocurvature fraction. On the other hand, we

TABLE I. The one-dimensional $2 \sigma$ ranges on the isocurvature mode coefficients for the various models, uncorrelated (second column) and correlated (third and fourth columns).

\begin{tabular}{cccc}
\hline \hline Model & $\alpha$ & $\alpha$ & $2 \beta[\alpha(1-\alpha)]^{1 / 2}$ \\
\hline CDI & $<0.31$ & $<0.47$ & -0.31 to 0.31 \\
BI & $<0.91$ & $<0.95$ & -0.80 to 1.00 \\
NID & $<0.76$ & $<0.77$ & -0.30 to 0.78 \\
NIV & $<0.60$ & $<0.60$ & -0.77 to 0.35 \\
\hline \hline
\end{tabular}



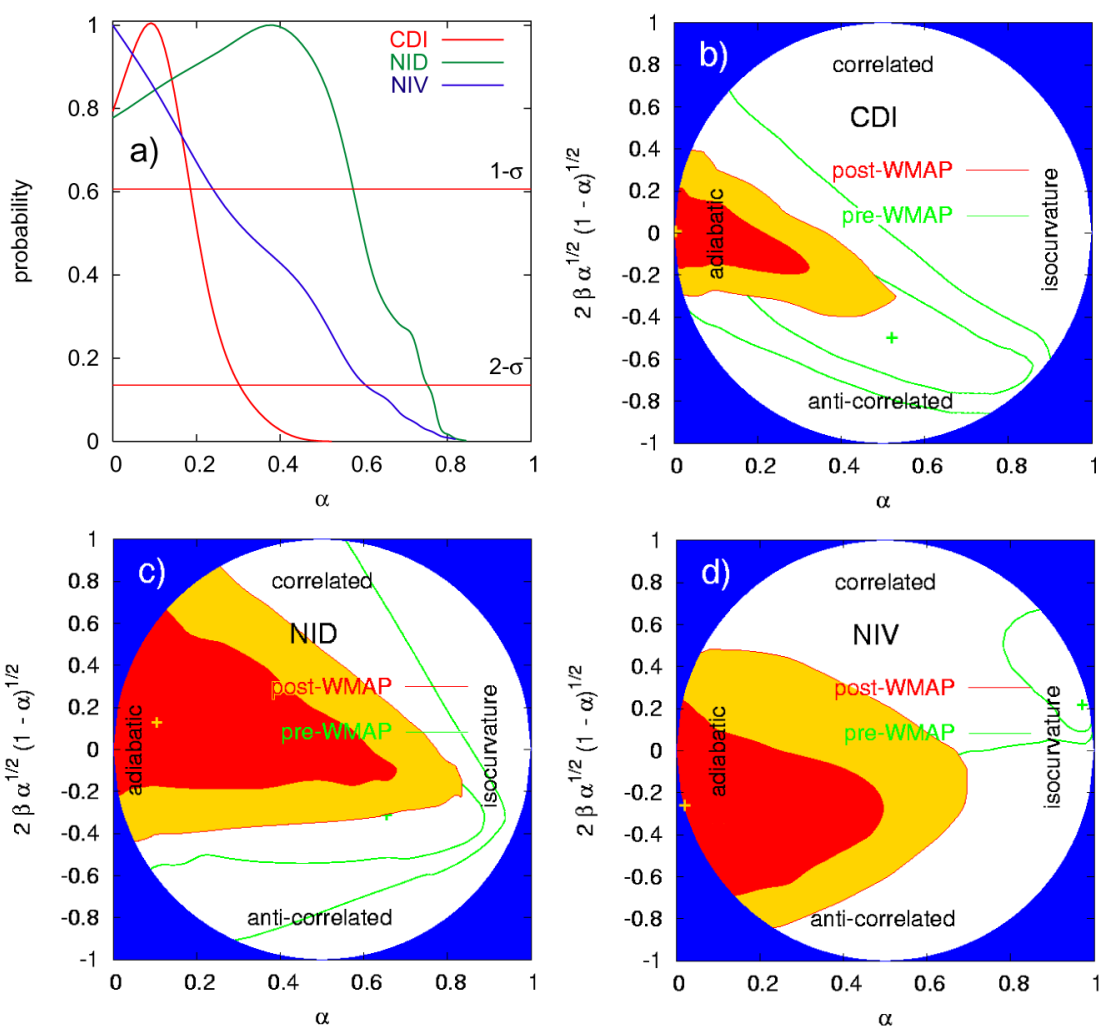

FIG. 1 (color online). (a) The likelihood function of the isocurvature fraction $\alpha$, for three different types of uncorrelated isocurvature modes (i.e., with the prior $\beta=0$ ); (b) the $1 \sigma$ and $2 \sigma$ contours of $\alpha$ and the cross-correlated mode coefficient $2 \beta \sqrt{\alpha(1-\alpha)}$, for the CDM isocurvature mode: the small contours are based on all the data, with one flat prior $n_{\text {iso }}>0.6$, while the large ones show the situation before WMAP, with an additional prior $\omega_{B}<0.037$; (c) same as (b) for NID; (d) same as (b) for NIV. The marginalization is approximated by a maximum likelihood fit of the other parameters for each pair of values $(\alpha, \beta)$. checked explicitly that the effect of the WMAP TE power spectrum is not very strong with respect to that of TT.

In the curvaton scenario, the case in which the CDM is created before the curvaton decay while the curvature perturbations are small, corresponding to $(\alpha, \beta)=$ $(0.9,-1)$ in our notation (3), is completely excluded, as already emphasized in Ref. [15]. The case in which the CDM is created by the decay of the curvaton leads to $\beta=1$; with such a prior, we obtain a sharp $2 \sigma$ bound: $\alpha<0.04$ or, in the notation of Ref. [14], $B<0.2$ (the authors of Ref. [15] obtain a more restrictive bound $B<$ $0.43 \Omega_{\mathrm{B}} / \Omega_{\mathrm{M}} \sim 0.1$, presumably because they use the curvaton-motivated assumption $n_{\text {iso }}=n_{\mathrm{ad}}$ ). We can rewrite this bound as a constrain on the fraction $r=$ $\Omega_{\sigma \text {, decay }}$ since, in these models, $B=3(1-r) / r$. In our case, $B<0.2$ implies $1-r<0.0625$.
Baryon isocurvature. - The case of baryon isocurvature modes is qualitatively similar to that of CDI modes, since the spectra are simply rescaled by a factor $\Omega_{\mathrm{B}}^{2} / \Omega_{\mathrm{cdm}}^{2}$ $\left(\Omega_{\mathrm{B}} / \Omega_{\mathrm{cdm}}\right)$ for the isocurvature power (cross-correlation) components: thus, significantly larger values of $\alpha$ will be allowed in the baryon case. Some approximate results for the BI modes could be deduced from the CDI results by an overall rescaling of the $\alpha$ parameter. However, we performed an exact analysis and found the following bounds: $\alpha<0.91$ (uncorrelated case), or $\alpha<0.95$ and $-0.8<$ $2 \beta \sqrt{\alpha(1-\alpha)}<1$ (correlated case). In each case, the minimum $\chi^{2}$ is, by definition, the same as for the CDI case. We provide the best-fit parameters in Table I, but for brevity we do not show this case in the figures.

In the curvaton scenario, the case in which the baryon number is created before the curvaton decay while the

TABLE II. The best fit values of the parameters for the adiabatic (AD), uncorrelated (CDI, etc.), and correlated (c-CDI, etc.) isocurvature models, with the corresponding $\chi^{2}$ and number of degrees of freedom $\nu$. For the NIV uncorrelated model, the best fit occurs for $\alpha=0$ (i.e., purely adiabatic).

\begin{tabular}{|c|c|c|c|c|c|c|c|c|}
\hline Model & $\omega_{\mathrm{B}}$ & $\omega_{\mathrm{cdm}}$ & $\Omega_{\Lambda}$ & $n_{\mathrm{ad}}$ & $n_{\text {iso }}$ & $\alpha$ & $\beta$ & $\chi^{2} / \nu$ \\
\hline $\mathrm{AD}$ & 0.021 & 0.12 & 0.70 & 0.95 & $\ldots$ & $\cdots$ & $\cdots$ & $1478.8 / 1435$ \\
\hline CDI & 0.023 & 0.12 & 0.73 & 0.99 & 1.02 & 0.10 & $\cdots$ & $1478.3 / 1433$ \\
\hline $\mathrm{BI}$ & 0.023 & 0.12 & 0.73 & 0.99 & 1.02 & 0.72 & $\ldots$ & $1478.3 / 1433$ \\
\hline NID & 0.023 & 0.12 & 0.73 & 0.99 & 0.95 & 0.37 & $\cdots$ & $1478.2 / 1433$ \\
\hline NIV & 0.021 & 0.12 & 0.70 & 0.95 & $\cdots$ & 0 & $\cdots$ & $1478.8 / 1433$ \\
\hline c-CDI & 0.022 & 0.12 & 0.71 & 0.97 & 1.23 & 0.001 & 1 & $1478.0 / 1432$ \\
\hline $\mathrm{c}-\mathrm{BI}$ & 0.022 & 0.12 & 0.71 & 0.97 & 1.23 & 0.03 & 1 & $1478.0 / 1432$ \\
\hline c-NID & 0.022 & 0.12 & 0.73 & 0.97 & 1.04 & 0.10 & 0.26 & $1477.7 / 1432$ \\
\hline c-NIV & 0.021 & 0.12 & 0.71 & 0.95 & 0.71 & 0.03 & -1 & $1477.5 / 1432$ \\
\hline
\end{tabular}


curvature perturbations are small, corresponding to $(\alpha, \beta)=(0.9,-1)$, is also excluded at several $\sigma$ 's, as previously found in Ref. [15]. The case in which the baryon number is created by the decay of the curvaton predicts $\beta=1$; we then find $\alpha<0.5$, or $B<1$.

Neutrino density isocurvature. - The $2 \sigma$ bound for the uncorrelated case is $\alpha<0.76$, while for the correlated adiabatic and neutrino modes we obtain $\alpha<0.77$ and $-0.30<2 \beta \sqrt{\alpha(1-\alpha)}<0.78$. The best-fitting models for the two cases are still extremely close to the adiabatic model, leading to no significant improvement in the likelihood, $\Delta \chi^{2}=0.6$ vs $\Delta \chi^{2}=1.1$, respectively. Note that with the pre-WMAP CMB data plus the 2dFGRS power spectrum, significantly larger fractions, up to $(\alpha, \beta)=$ $(0.9,-0.6)$, of correlated NID modes were then allowed, but are now excluded with great confidence.

The authors of Ref. [8] discuss an interesting mechanism under which the curvaton could generate some fully correlated or anticorrelated NID modes, through inhomogeneities in the neutrino/antineutrino asymmetry parameter. However, our bounds for these cases are very restrictive, $\alpha<0.18$ (correlated), or $\alpha<0.02$ (anticorrelated). In the notation of Ref. [14], this is equivalent to $B<0.47$ or $B<0.14$, respectively.

Neutrino velocity isocurvature. - Again, the presence of such a mode is not significantly favored: in the uncorrelated case, the best-fitting model is still the pure adiabatic one $(\alpha=0)$, while in the correlated case the $\chi^{2}$ improvement is only $\Delta \chi^{2}=1.3$. The impact of WMAP on these bounds is spectacular. Using only the pre-WMAP CMB data compilation and the 2dFGRS power spectrum, we find that half of the parameter space (with $0<\alpha<1$ and $0<\beta<1$ ) was allowed until recently (at the expense of an unnaturally high baryon fraction and scalar tilt).

Conclusions. - Using the recent measurements of temperature and polarization anisotropies in the CMB by WMAP, as well as the matter power spectrum measured by $2 \mathrm{dFGRS}$, we obtained very stringent bounds on a possible isocurvature component in the primordial spectrum of density and velocity fluctuations. We have considered both correlated and uncorrelated adiabatic and isocurvature modes and put strong constraints on the curvaton scenario of primordial perturbations.

[1] A. D. Linde, Phys. Lett. B158, 375 (1985); L. A. Kofman and A. D. Linde, Nucl. Phys. B282, 555 (1987); S. Mollerach, Phys. Lett. B 242, 158 (1990); A. D. Linde and V. Mukhanov, Phys. Rev. D 56, 535 (1997); M. Kawasaki, N. Sugiyama, and T. Yanagida, Phys.
Rev. D 54, 2442 (1996); P. J. E. Peebles, Astrophys. J. 510, 523 (1999).

[2] D. Polarski and A. A. Starobinsky, Phys. Rev. D 50, 6123 (1994); M. Sasaki and E. D. Stewart, Prog. Theor. Phys. 95, 71 (1996); J. García-Bellido and D. Wands, Phys. Rev. D 53, 5437 (1996); M. Sasaki and T. Tanaka, Prog. Theor. Phys. 99, 763 (1998).

[3] C. Gordon, D. Wands, B. A. Bassett, and R. Maartens, Phys. Rev. D 63, 023506 (2001); N. Bartolo, S. Matarrese, and A. Riotto, Phys. Rev. D 64, 123504 (2001); D. Wands, N. Bartolo, S. Matarrese, and A. Riotto, Phys. Rev. D 66, 043520 (2002); F. Di Marco, F. Finelli, and R. Brandenberger, Phys. Rev. D 67, 063512 (2003).

[4] D. Langlois, Phys. Rev. D 59, 123512 (1999); D. Langlois and A. Riazuelo, Phys. Rev. D 62, 043504 (2000); S. Tsujikawa, D. Parkinson, and B. A. Bassett, Phys. Rev. D 67, 083516 (2003).

[5] G. Efstathiou and J. R. Bond, Mon. Not. R. Astron. Soc. A 218, 103 (1986); 227, 33 (1987); P. J. E. Peebles, Nature (London) 327, 210 (1987); H. Kodama and M. Sasaki, Int. J. Mod. Phys. A 1, 265 (1986); 2, 491 (1987); S. Mollerach, Phys. Rev. D 42, 313 (1990).

[6] A. K. Rebhan and D. J. Schwarz, Phys. Rev. D 50, 2541 (1994); M. Bucher, K. Moodley, and N. Turok, Phys. Rev. D 62, 083508 (2000); Phys. Rev. Lett. 87, 191301 (2001).

[7] D. H. Lyth and D. Wands, Phys. Lett. B 524, 5 (2002); T. Moroi and T. Takahashi, Phys. Lett. B 522, 215 (2001); Phys. Rev. D 66, 063501 (2002).

[8] D. H. Lyth, C. Ungarelli, and D. Wands, Phys. Rev. D 67, 023503 (2003).

[9] The WMAP Collaboration, C. L. Bennett et al., Astrophys. J. Suppl. Ser. 148, 1 (2003); L. Page et al., astro-ph/0302220; H. V. Peiris et al., Astrophys. J. Suppl. Ser. 148, 213 (2003).

[10] The ACBAR Collaboration, C. L. Kuo et al., astro-ph/ 0212289; J. H. Goldstein et al., astro-ph/0212517.

[11] The 2dFGRS Collaboration, J. A. Peacock et al., Nature (London) 410, 169 (2001); W. J. Percival et al., Mon. Not. R. Astron. Soc. A 327, 1297 (2001); 337, 1068 (2002).

[12] R. Stompor, A. J. Banday, and K. M. Gorski, Astrophys. J. 463, 8 (1996); P. J. E. Peebles, Astrophys. J. 510, 531 (1999); E. Pierpaoli, J. García-Bellido, and S. Borgani, J. High Energy Phys. 9910 (1999) 015; M. Kawasaki and F. Takahashi, Phys. Lett. B 516, 388 (2001); K. Enqvist, H. Kurki-Suonio, and J. Väliviita, Phys. Rev. D 62, 103003 (2000); 65, 043002 (2002).

[13] R. Trotta, A. Riazuelo, and R. Durrer, Phys. Rev. Lett. 87, 231301 (2001); Phys. Rev. D 67, 063520 (2003).

[14] L. Amendola, C. Gordon, D. Wands, and M. Sasaki, Phys. Rev. Lett. 88, 211302 (2002).

[15] C. Gordon and A. Lewis, Phys. Rev. D 67, 123513 (2003).

[16] J. Väliviita and V. Muhonen, Phys. Rev. Lett. 91, 131302 (2003).

[17] http://www.hep.upenn.edu/ max/cmblsslens.html; a previous version is described in X. Wang, M. Tegmark, B. Jain, and M. Zaldarriaga, astro-ph/0212417. 